\title{
ChemComm
}

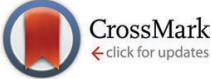

Cite this: Chem. Commun., 2016, 52, 13385

Received 22nd September 2016, Accepted 18th October 2016

DOI: $10.1039 / c 6 c c 07714 e$

www.rsc.org/chemcomm

\section{Following the stability of amphiphilic nanoaggregates by using intermolecular energy transfer $\dagger$}

\author{
M. Harris, $\ddagger^{\mathrm{a}}$ H. De Keersmaecker, $\ddagger^{\mathrm{b}}$ L. Vander Elst, ${ }^{\mathrm{cd}}$ E. Debroye, ${ }^{a}$ Y. Fujita, ${ }^{a}$ \\ H. Mizuno ${ }^{b}$ and T. N. Parac-Vogt*a
}

\begin{abstract}
An intermolecular energy transfer system is developed for studying the stability of nanoaggregate(s) (NAs) in complex solution and cell culture by one- and two-photon fluorescence microscopy and optical imaging. The system allows facile addition of one or more tumor targeting molecules, one of which is exemplified here. NAs functionalized with an MRI and optical probe, with and without folic acid, remain stable in fetal bovine serum for at least 4 hours. HeLa cell cultures showed a clear difference between NAs nontargeted and targeted to folate receptors, with both NAs appearing to be taken up by the cells through different mechanisms. An MRI relaxivity, $r_{1}$, of $9 \mathrm{mM}^{-1} \mathrm{~s}^{-1}$ at $310 \mathrm{~K}$ and $1.4 \mathrm{~T}$ was measured associated with the increased rotational correlation time of the NAs. These NAs may have application in the targeted drug delivery of hydrophobic drugs such as doxorubicin (DOX).
\end{abstract}

Nanomedicine and targeted drug delivery are important multidisciplinary fields encompassing chemical, pharmaceutical, and medical research striving to improve the efficacy and minimize the side effects of current therapeutic treatments, particularly chemotherapy. ${ }^{1}$ For this application, inorganic nanoparticles (NPs) ${ }^{2}$ and polymeric micelles (PMs) ${ }^{3}$ have been intensely studied as drug delivery systems by loading these particles with appropriate drugs. Disadvantages of these systems are the toxicity concern regarding $\mathrm{NPs}^{4}$ and the limited number of examples of assessing the stability of PMs. ${ }^{5}$ Further progress requires the active targeting of tumors, with significantly

\footnotetext{
${ }^{a}$ Department of Chemistry, KU Leuven, 3001 Leuven, Belgium.

E-mail: tatjana.vogt@kuleuven.be

${ }^{b}$ Department of Chemistry, Biochemistry, Molecular and Structural Biology Section, Laboratory of Biomolecular Network Dynamics, KU Leuven, 3001 Leuven, Belgium ${ }^{c}$ Department of General, Organic and Biomedical Chemistry, NMR and Molecular Imaging Laboratory, University of Mons, 7000 Mons, Belgium

${ }^{d}$ CMMI - Centre for Microscopy and Molecular Imaging, 6041 Gosselies, Belgium $\dagger$ Electronic supplementary information (ESI) available: Experimental details; characterisation of ligands and complexes; ESI-MS spectra of the complexes; and dynamic light scattering profiles of the NA solutions. See DOI: 10.1039/ c6cc07714e

\$ Authors M. Harris and H. De Keersmaecker contributed equally.
}

decreased non-specific side effects. This approach is typically performed by the surface conjugation of drug carriers to targeting molecules, such as antibodies, ${ }^{6}$ peptides, ${ }^{7}$ or molecules targeting over-expressed receptors in tumor cells such as transferrin $^{8}$ and folate. ${ }^{9}$

PMs are core/shell particles of amphiphilic block copolymers with sizes ranging from 5 to $100 \mathrm{~nm}$. It has been reported that folic acid (FA)-conjugated DOX-loaded PMs exhibit potent cytotoxicity against folate receptor-expressing human ovarian cancer cells in vitro. ${ }^{10}$ However, very little is known with regard to the efficacy of these PMs in human tumors. Preliminary studies of the stability of these systems in different media, such as human serum and cell culture, are an important first step prior to in vivo testing.

Here, we report a new approach to follow the stability of mixed NAs of a phospholipid (DPPC), stabilized with the surfactant Tween $80^{\mathbb{R}}$. Into this nanoaggregate system, an amphiphilic $\mathrm{Gd}^{3+}$ MRI chelate bearing a fluorescent chromophore (energy donor) is incorporated with a $\mathrm{Eu}^{3+}$ optical chelate (energy acceptor) (Fig. 1). Additionally, folic acid was introduced into the NAs, modified by adding two alkyl chains. The aggregate was investigated both for its in vitro stability

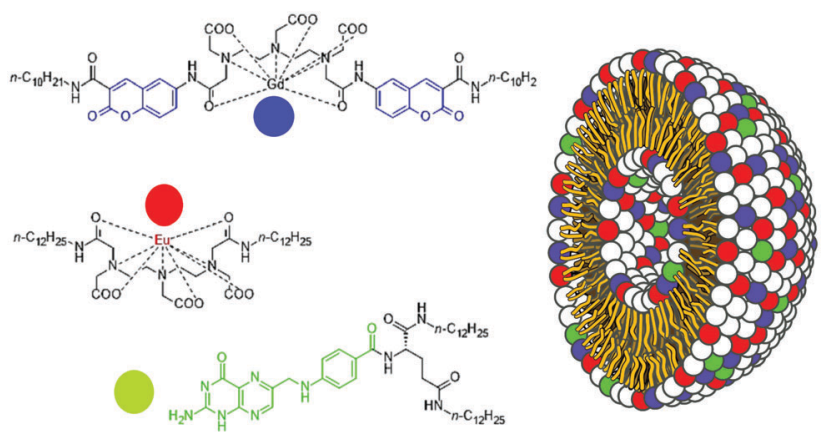

Fig. $1 \mathrm{Gd}($ III)-DTPA-BC 10 coumarin donor (blue), Eu(III)-DTPA-BC 12 acceptor (red), folate- $\mathrm{BC}_{12}$ target (green) and schematic of the nanoaggregate. In the lanthanide chelates, the coordinated water molecule and DPPC (white) have been omitted. 
and the cellular uptake mechanism in HeLa cells addressed in both non- and targeted NAs, aided by the high expression of folate receptors on the surface of these particular cancer cells. ${ }^{11}$ Indeed, any active targeting molecule can be mixed with the system, provided that it is amphiphilic, for example, having alkyl chains present or added. The idea of loading the hydrophobic cavity with chromophores has been previously reported for sensitizing lanthanide luminescence using $\mathrm{Eu}^{3+}$ or NIR emitting lanthanide ions. ${ }^{12}$ However, to the best of our knowledge, this is the first example of a receptor-targeted system where the donor and acceptor are positioned on the surface, leaving the inner cavity (micelles) or bilayer (liposomes) free for drug loading. In this case, both MRI and optical methods can be applied to monitor the stability and biodistribution.

Mixed NAs consisting of $\mathrm{Gd}(\mathrm{III})-\mathrm{DTPA}-\mathrm{BC}_{10}$ coumarin donor/ Eu(III)-DTPA-chelate acceptor, with and without folate, were prepared with homogeneous size distributions of 63 and $58 \mathrm{~nm}$, respectively (ESI, $\dagger$ Fig. S4 and S5). The molar ratio of the various components was $3: 1: 1: 2$ of phospholipid: donor : acceptor: surfactant, with 0.5 equivalents of modified folate added to the targeted NAs. The critical micelle concentration (cmc) of DPPC is reported to be $0.46 \mathrm{nM}^{13}$ which is 5 orders of magnitude lower than the most dilute concentration $(350 \mu \mathrm{M})$ used in cell culture incubation studies in this work. The increased relaxivity of the $\mathrm{Gd}^{3+}$ conjugate of $9 \mathrm{mM}^{-1} \mathrm{~s}^{-1}$ $(60 \mathrm{MHz}, 310 \mathrm{~K})$ is a significant increase compared to a value of $3.5 \mathrm{mM}^{-1} \mathrm{~s}^{-1}$ for Gd(III)-DTPA (Magnevist ${ }^{\circledR}$ ), which corresponds to the DTPA chelate being aggregated into NAs. The coumarin used in this system exhibits a polarity sensitive excitation spectrum. For further investigation of the environment of the chromophore, we studied the excitation spectrum in solvents of various polarities to confirm that it is aggregated and not free in solution. When the excitation spectrum is compared while monitoring the coumarin emission at $450 \mathrm{~nm}$, the spectrum resembles the spectra taken in DMSO and varies significantly from that observed in water, suggesting that the coumarin is not located in an aqueous environment, but is aggregated within the surface layer of the NAs (ESI, $\dagger$ Fig. S6).

In vitro excitation of NAs with and without folate at $370 \mathrm{~nm}$ led to the characteristic europium emission bands, due to the ${ }^{5} \mathrm{D}_{0} \rightarrow{ }^{7} \mathrm{~F}_{J}(J=0-4)$ transitions, with the $615 \mathrm{~nm}$ transition being the most intense (Fig. 2). Such behavior confirms the proximity of the donor and acceptor pair, allowing intermolecular energy transfer for sensitized lanthanide luminescence. This phenomenon is observed when the energy donor and acceptor are in close proximity $(\sim 5 \AA)$. When the donor and acceptor are mixed together in a solution of chloroform and methanol $(1: 1)$, the coumarin fluorescence dominates, consistent with the donor and acceptor being not aggregated, leading to inefficient energy transfer (ESI, $\dagger$ Fig. S8).

NAs of only Gd(III)-DTPA-BC 10 coumarin/DPPC or Eu(III)-DTPA$\mathrm{BC}_{12} / \mathrm{DPPC}$ show coumarin fluorescence or very weak $\mathrm{Eu}^{3+}$ direct excited emission, confirming that an intense red emission could only be attributable to the proposed energy transfer process (ESI, $\dagger$ Fig. S9). However, when the two molecules are mixed in aqueous solution with the phospholipid DPPC and Tween $80^{\circledR}$, the $\mathrm{Eu}^{3+}$

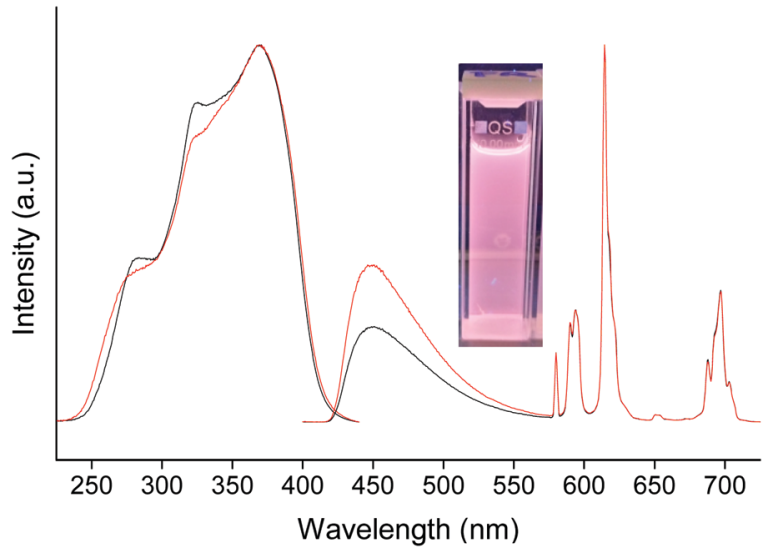

Fig. 2 Normalised excitation and emission spectra of Gd(III)-DTPA$\mathrm{BC}_{10}$ coumarin/Eu(II)-DTPA-BC 12 (black) and Gd(II)-DTPA-BC 10 coumarin/ $\mathrm{Eu}(\mathrm{III})-\mathrm{DTPA}-\mathrm{BC}_{12} /$ folate- $\mathrm{BC}_{12}$ (red) in DPPC NAs (water, $10^{-4} \mathrm{M}, 298 \mathrm{~K}$ ). Cuvette showing red luminescence (inset). The excitation wavelength is $370 \mathrm{~nm}$ and the excitation spectrum is taken while monitoring emission at $615 \mathrm{~nm}$.

luminescence dominates, suggesting aggregation and the proximity of the donor and acceptor (Fig. 2). Moreover, a two-photon excited emission (2PE) was observed using an excitation wavelength of $800 \mathrm{~nm}$ (Ti:sapphire laser) (Fig. 3). Such 2PE emission is of particular interest for in vitro studies due to enhanced depth discrimination, and for in vivo studies due to the increased optical transparency of tissues to longer wavelength light and a reduction of photo-damage. ${ }^{14}$

In aqueous solution, long luminescence lifetimes of 0.95 and $0.96 \mathrm{~ms}( \pm 0.01)$ were observed for non-folate and folate NAs respectively. A luminescence lifetime in the ms range is of particular interest for time-gated luminescence studies to avoid biological autofluorescence. ${ }^{15}$ The relative quantum yield to rhodamine 101 in ethanol under ligand excitation $\left(Q_{\mathrm{Eu}}^{\mathrm{L}}\right)$ was $1 \%$ $( \pm 0.1)$ and addition of folate to the NAs showed no effect. However, due to the sharp emission lines of $\mathrm{Ln}^{3+}$ ions, the relative quantum yield may be misleading in representing the quality of

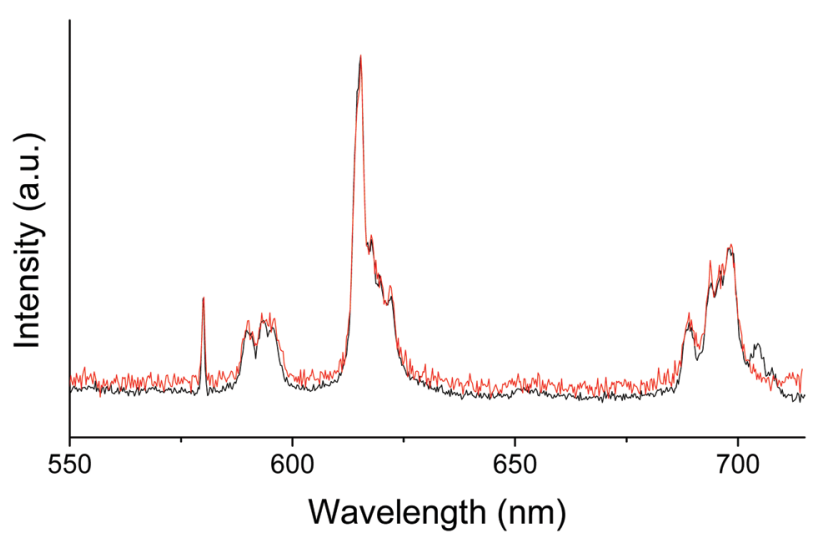

Fig. 3 Normalised 2PE spectra of Gd(III)-DTPA-BC 10 Coumarin/Eu(III)DTPA-BC 12 (black) and Gd(III)-DTPA-BC 10 coumarin/Eu(III)-DTPA-BC 12 / folate- $\mathrm{BC}_{12}$ (red) DPPC NAs (800 nm Ti:sapphire laser excitation, water, $\left.10^{-4} \mathrm{M}, 298 \mathrm{~K}\right)$ 
the luminescent probe, as it is performed using a comparative method of integrating the area below the spectrum of an organic dye standard, like rhodamine 101, which shows a very broad emission. For $\mathrm{Ln}^{3+}$ probes, peak intensity is the most important parameter and in the $\mathrm{Ln}$ series, $\mathrm{Eu}^{3+}$ is known for its intense red emission.

We have recently investigated the stability of MRI contrast agent mixed micelle systems based on DPPC using dynamic light scattering (DLS), and found that there was no change in the micelles when incubated at $37{ }^{\circ} \mathrm{C}$ for 1 week. ${ }^{16}$ However, the major drawback of the DLS method is that it cannot be used for a complex mixture of globular proteins. Redesigning our previous systems by placing the donor chromophore and acceptor $\mathrm{Eu}^{3+}$ of the energy transfer system on separate molecules has made it possible to follow the stability of these NAs directly in fetal bovine serum (FBS), a complex solution with biologically relevant ionic strength. When incubated at $37{ }^{\circ} \mathrm{C}$ in FBS, the NAs without folate showed a slow decrease in luminescence intensity over 24 hours (ESI, $\dagger$ Fig. S7). When folate is present, there is a similar luminescence loss observed and the decreasing 615/450 nm peak ratio is visible in the spectrum with the sample becoming visually more blue fluorescent (Fig. 4).

For fluorescence microscopy, excitation in the tail region of the donor chromophore at $405 \mathrm{~nm}$ was used for studies in cultured HeLa cells (Fig. 5). The NAs without folate were taken up by the cells and showed an intense signal in the acceptor channel (Fig. 5C). With folate receptor targeted NAs, a difference was observed in a decreased acceptor emission (Fig. 5D), indicating either reduced energy transfer efficiency and/or changing uptake efficiencies.

Reduction of the energy transfer efficiency might be caused by dispersion of the NAs. Such behavior suggests that the NAs are taken into the cells via an alternative pathway, most likely the folate receptor mediated endocytosis. Additionally, cell culture was performed with only donor NAs and only acceptor NAs to

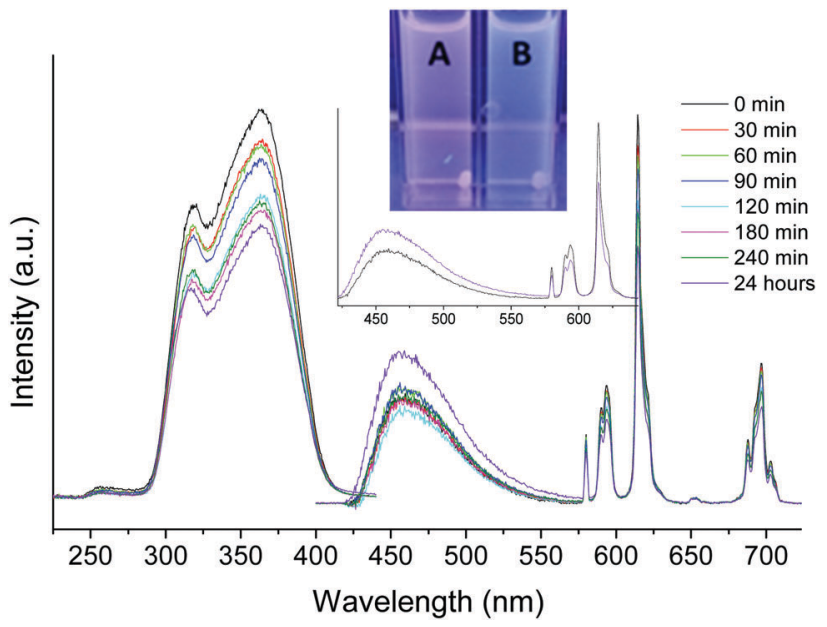

Fig. 4 Excitation and emission spectra of 1:1 FBS with Gd(II)-DTPA$\mathrm{BC}_{10}$ coumarin/Eu(II)-DTPA-BC 12 /folate DPPC NAs (FBS background subtracted, excitation at $370 \mathrm{~nm}$ and excitation spectrum taken while monitoring $615 \mathrm{~nm}$, water, $\left.10^{-4} \mathrm{M}, 298 \mathrm{~K}\right)$. Non-folate NAs after 24 hours (A) and folate NAs after 24 hours (B).
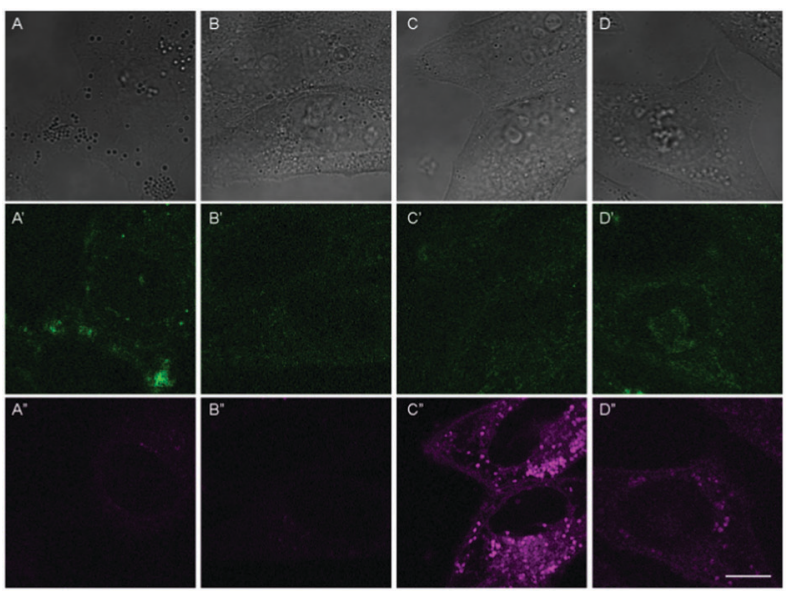

Fig. 5 DIC images (grey) and fluorescence images of the donor channel (green) and acceptor channel (magenta) for only donor NAs (A), only acceptor NAs (B), NAs without folate (C) and folate receptor targeted $\mathrm{NAs}$ (D) after excitation with a $405 \mathrm{~nm}$ laser diode measured with confocal laser scanning microscopy; scale bar indicates $10 \mu \mathrm{m}$; concentration was $0.35 \mathrm{mM}$.

confirm that the signal was obtained from the donor/acceptor system. Blue emission could be observed coming from the coumarin chromophore but with a very low intensity (Fig. $5 \mathrm{~A}^{\prime}$ ). NAs with only $\mathrm{Eu}^{3+}$ showed minimal emission, consistent with the hypothesis that the signals in the acceptor channel are caused by intermolecular energy transfer.

The viability of HeLa cells was evaluated in the presence of NAs by an MTT assay based on perturbation of mitochondrial

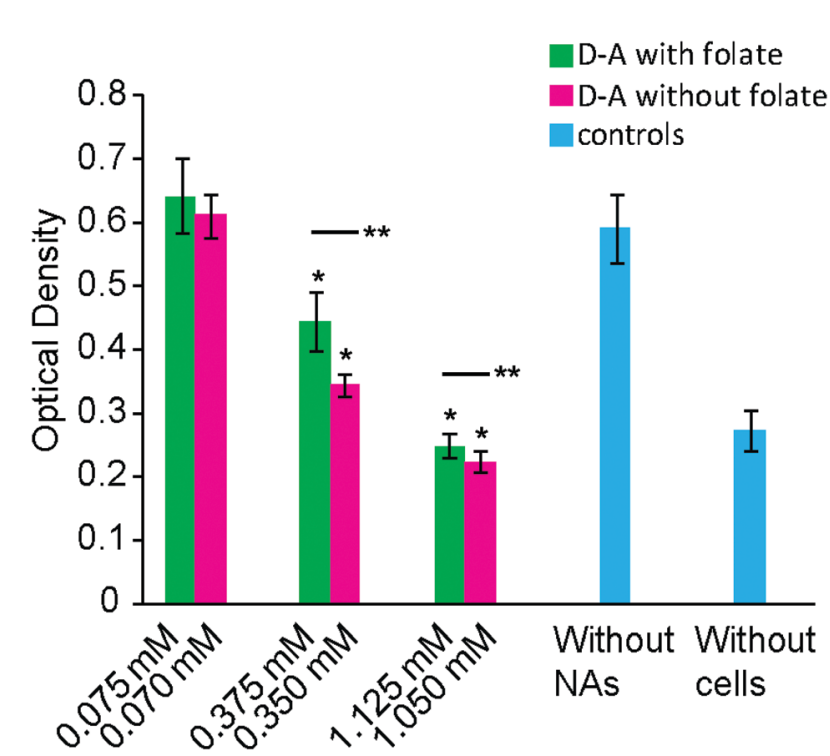

Fig. 6 Optical density for the MTT assay after addition of NAs to HeLa cells for both NAs without folate (magenta) and folate receptor targeted NAs (green). As a reference, the MTT assay for control samples without addition of NAs to the cells and the MTT assay without cells or NAs are shown (cyan). Significant difference was observed between $0.375 / 0.35$ or 1.125/1.05 mM NA concentration and no NA addition (*) as well as between NAs with and without folate for a $0.375 / 0.35$ and 1.125/1.05 mM concentration ${ }^{* *}$ ) with a $P$ value of $<0.05$. 
redox activity (Fig. 6). The cell proliferation was completely prohibited in the presence of $\sim 1 \mathrm{mM}$ NAs regardless of targeting to the folate receptor, whereas no significant effect was observed at $0.07 \mathrm{mM}$. At $\sim 0.35 \mathrm{mM}$, proliferation was limited to about $20 \%$ or $50 \%$ with or without targeting to folate receptors, respectively, indicating lower cytotoxicity of NAs targeted to folate receptors than non-targeted NAs.

In summary, a novel intermolecular energy transfer system of mixed NAs with and without a targeting group has been synthesised and evaluated for its stability and potential as a drug delivery system. Cell culture studies were carried out with HeLa cells with overnight incubation. Without folate, the system showed a large accumulation of NAs taken up by the cells and an intense acceptor signal being emitted, suggesting that the NAs are in high local concentration within the endosomes and are able to exhibit strong emission. When folate is added, the acceptor signal decreases, which suggests that a receptor pathway is utilized and the NAs disperse in the cell or a lower amount of NAs is taken up. We note in relation to the cytotoxicity results that the current nano-aggregate formulations were not optimised for biological purposes. This proof-ofconcept study shows that a chromophore donor, lanthanide acceptor/emitter, and targeting molecule can be integrated into the supramolecular structure to follow the stability of the object by photophysical techniques. For optical applications, the NAs can be excited by one- or two-photon processes. Monitoring emission from intermolecular energy transfer is shown to be an elegant and novel method for interpreting the stability of the NAs in vitro. By adding folate as a targeting vector, a different cellular uptake pathway is presented, which is of particular interest for drug loading, especially the targeted delivery of hydrophobic drugs.

The simplicity with which the system can be modified to carry any targeting molecule, which only needs alkyl chain functions, is a major advantage allowing the targeting of any tumor for which a cell-surface receptor has been identified. The nanoaggregate system has a final 'luxury' property, allowing potential incorporation of multiple imaging modalities into one nano-object. In this work we included the MRI positive contrast agent based on $\mathrm{Gd}^{3+}$, but inclusion of a modified radionuclide chelate could allow PET to be used as an additional imaging modality.

We acknowledge financial support from the Research Foundation-Flanders (FWO Flanders, Belgium) for a doctoral fellowship granted to $\mathrm{M}$. H. and a postdoctoral fellowship granted to E. D. and H. D. K. is supported by a PhD grant
Agentschap voor Innovatie door Wetenschap en Technologie (IWT number 121165) of the Institute for the Promotion of Innovation through Science and Technology in Flanders (IWT-Vlaanderen). Mass spectrometry was made possible by the support of the Hercules Foundation of the Flemish Government (grant 20100225-7).

\section{Notes and references}

1 (a) S. S. Linton, S. G. Sherwood, K. C. Drews and M. Kester, Wiley Interdiscip. Rev.: Nanomed. Nanobiotechnol., 2016, 8, 208-222; (b) A. M. d. P. Bayona, C. M. Moore, M. Loizidou, A. J. MacRobert and J. H. Woodhams, Int. J. Cancer, 2015, 138, 1049-1057; (c) R. B. Campbell, B. Ying, G. M. Kuesters and R. Hemphill, J. Pharm. Sci., 2008, 98, 411-429.

2 (a) J. Shen, L. Zhao and G. Han, Adv. Drug Delivery Rev., 2013, 65, 744-755; (b) T. Doane and C. Burda, Adv. Drug Delivery Rev., 2013, 65, 607-621; (c) S. Bhattacharyya, R. A. Kudgus, R. Bhattacharya and P. Mukherjee, Pharm. Res., 2011, 28, 237-259; (d) C. E. Probst, P. Zrazhevskiy, V. Bagalkot and X. Gao, Adv. Drug Delivery Rev., 2012, 65, 703-718.

3 (a) K. J. Haxton and H. M. Burt, J. Pharm. Sci., 2009, 98, 2299-2316; (b) M. L. Adams, A. Lavasanifar and G. S. Kwon, J. Pharm. Sci., 2003, 92, 1343-1355; (c) S. Movassaghian, O. M. Merkel and V. P. Torchilin, Wiley Interdiscip. Rev.: Nanomed. Nanobiotechnol., 2015, 7, 691-707; (d) K. S. Ho and M. S. Shoichet, Curr. Opin. Chem. Eng., 2013, 2, 53-59. 4 (a) R. Podila and J. M. Brown, J. Biochem. Mol. Toxicol., 2012, 27, 50-55; (b) A. Albanese, P. S. Tang and W. C. W. Chan, Annu. Rev. Biomed. Eng., 2012, 14, 1-16.

5 (a) J. Lu, S. C. Owen and M. S. Shoichet, Macromolecules, 2011, 44, 6002-6008; (b) C. L. Lo, C. K. Huang, K. M. Lin and G. H. Hsiue, Biomaterials, 2007, 28, 1228-1235; (c) Y. P. Li, S. R. Pan, W. Zhang and Z. Du, Nanotechnology, 2009, 20, 1-11; (d) H. Chen, S. Kim, W. He, H. Wang, P. S. Low, K. Park and J. X. Cheng, Langmuir, 2008, 24, 5213-5217; (e) S. Jiwpanich, J. H. Ryu, S. Bickerton and S. Thayumanavan, J. Am. Chem. Soc., 2010, 132, 10683-10685.

6 E. L. Sievers and P. D. Senter, Annu. Rev. Med., 2013, 64, 15-29.

7 (a) A. Accardo and G. Morelli, J. Pept. Sci., 2015, 104, 462-479; (b) S. Majumdar and T. J. Siahaan, Med. Res. Rev., 2012, 32, 637-658. 8 H. Li and Z. M. Qian, Med. Res. Rev., 2002, 22, 225-250.

9 A. R. Hilgenbrink and P. S. Low, J. Pharm. Sci., 2005, 94, 2135-2146. 10 (a) S. Panjaa, S. Majib, T. K. Maitib and S. Chattopadhyay, Polymer, 2015, 61, 75-86; (b) C. Yua, C. Gaoa, S. Lüa, C. Chena, J. Yanga, X. Dib and M. Liu, Colloids Surf., B, 2014, 115, 331-339.

11 C. P. Leamon and P. S. Low, Biochem. J., 1993, 291, 855-860.

12 (a) S. Lacerda, C. S. Bonnet, A. Pallier, S. Villette, F. Foucher, F. Westall, F. Buron, F. Suzenet, C. Pichon, S. Petoud and É. Tóth, Small, 2013, 9, 2662-2666; (b) C. S. Bonnet, L. Pellegatti, F. Buron, C. M. Shade, S. V. V. Kubícek, G. G. F. S. S. Petoud and É. Tóth, Chem. Commun., 2010, 46, 124-126.

13 Product specification: Avanti Polar Lipids Inc., Product code: 850355.

14 (a) Y. Chen, R. Guan, C. Zhang, J. Huang, L. Ji and H. Chao, Coord. Chem. Rev., 2016, 310, 16-40; (b) T. Nemoto, R. Kawakami, T. Hibi, K. Iijima and K. Otomo, Microscopy, 2015, 64, 9-15.

15 (a) J. Vuojola and T. Soukka, Methods Appl. Fluoresc., 2014, 2, 1-28; (b) R. Cubeddu, D. Comelli, C. D'Andrea, P. Taroni and G. Valentini, J. Phys. D: Appl. Phys., 2002, 35, R61-R76.

16 M. Harris, L. V. Elst, S. Laurent and T. N. Parac-Vogt, Dalton Trans., 2016, 45, 4791-4801. 